\title{
Topological conditional entropy
}

by

\section{MICIAX MISIUREWICZ (Warszawa)}

Abstract. A new invariant of discrete dynamical systems - topological conditional entropy - is introduced. Its ranishing (i.e. asymptotical $h$-expansiveness) implies the upper semi-continuity of measure-theoretic entropy, regarded as a function of an invariant regular normed Borel measure (and in particular - the existence of a measure with maximal entropy). All the endomorphisms of compact topological groups are shown to be asymptotically $h$-expansive. Expansiveness or $h$-expansiveness groups are shown to be asymptotically $h$-exp
also imply asymptotical $h$-expansiveness.

A very short proof is given of the Goodwyn's theorem on the bounding of measuretheoretic entropy by topological entropy. Also a new proof is given of the formula for topological entropy of a product of transformations.

\$ 0. Introduction. In the present paper we consider continuous transformations of compact Hausdorff spaces into themselves (cascades). We define a new invariant, which te call 'topological conditional entropy'. It is based on the notion of topological entropy ([1]), and it is also close to the notions of $h$-expansiveness ([5]) and asymptotical $h$-expansiveness ([15]). Some of its properties are the same as those of topological entropy. Topological conditional entropy is also connected with measure-theoretic entropy, regarded as a function of an invariant regular normed Borel measure. Unfortunately, it cannot be defined by means of the above function, and therefore topological tools cannot be aroided in some proofs (contrary to some results concerning usual topological entropy admitting a proof by a reduction to simple measure-theoretic facts).

\$1. Definitions. We shall consider a continuous transformation $f: X \rightarrow X$ of a non-empty compact Hausdorff space $X$ into itself. Such a pair $(X, f)$ is called a casoade.

A sot $Y \subset X$ is called invariant (under $f$ ) if $f X \subset Y$; it is called strictly invariant if $f Y=Y$. If $(Z, g)$ is also a cascade, and if there exists a continuous surjection $p: X \rightarrow Z$ such that $g \circ p=p \circ f$, then $(Z, g)$ is called a factor of $(X, f)$.

Denote by $\mathscr{P}(X)$ the set of all covers of the space $X$ containing a finite subcover, and by $\mathfrak{A}(X)$ the set of all open finite covers of $X$ (we write simply $\mathscr{P}$ and $\mathfrak{A}$ if we consider only one space). 
For $Y \subset X, A \in \mathscr{P}$, write $Y \prec A$ if there exists an $a \in A$ such that $Y \subset a$. For $A, B \in \mathscr{P}$ write $A \geqslant B(A$ is a refinement of $B$ ) if $a \prec B$ for every $a \in A$. For a family $\left\{A_{i}\right\}_{i \in I}$, let

$$
\bigvee_{i \in I} A_{i}=\left\{\bigcap_{i \in I} a_{i}: a_{i} \in A_{i} \text { for } i \in I\right\}
$$

(or $A_{i_{1}} \vee \ldots \vee A_{i_{n}}$ for a finite set $I=\left\{i_{1}, \ldots, i_{n}\right\}$ ).

Card $I$ denotes the cardinality of the set $I$. If Card $I<\infty$ and $A_{i} \in \mathscr{P}$ (resp. $\mathfrak{U}$ ) for $i \in I$ then, of course, also $\bigvee_{i \in I} A_{i} \in \mathscr{P}$ (resp. $\mathfrak{X}$ ). The operation ' $\vee$ ' preserves the relation ' $\leqslant$ '. The sets $\mathscr{P}$ and $\mathfrak{A}$ are directed by the relation $\leqslant$. Clearly, for $A, B \in \mathscr{P}, A \vee B \geqslant A$. For $A \in \mathscr{P}, n \in \mathbb{Z}$, let $f^{n} A$ $=\left\{f^{n} a: a \in A\right\} ;$ for $n \in \boldsymbol{N}, A_{f}^{n}=\bigvee_{i=0}^{n-1} f^{-i} A$ (if we consider only one transformation, we write simply $A^{n}$ ).

For a non-empty set $Y \subset X$ and a cover $A \in \mathscr{P}$ write

$$
N(Y, A)=\min \{\text { Oard } O: O \subset A, \dot{Y} \subset \cup C\} .
$$

For the empty set put $N(\varnothing, A)=1$. For $A, B \in \mathscr{P}$ write

$$
N(A \mid B)=\max _{b \in B} \cdot N(b, A)
$$

Further, $N(A)=N(X, A)$. We write also $\Theta(X)=\{X\} \in \mathfrak{A}$ (if we consider only one space, we write simply $\Theta)$. Of course, we have

$$
\text { (1.1) } \quad N(A)=N(A \mid \Theta) \text { : for } A \in \mathscr{P} \text {. }
$$

Now we list the simplest properties of the function $N$, similar to the properties of the functions $\exp H$ for measure-theoretic entropy (see [2]).

Let $A, B, C, D \in \mathscr{P} ; Y, Z \subset X$. The following inequalities hold:

$$
\begin{aligned}
N(Y, A) \leqslant N(Z, B) & \text { for } \quad B \geqslant A, \quad Y \subset Z, \\
N(A \mid B) \leqslant N(C \mid D) \quad \text { for } \quad C \geqslant A, B \geqslant D, & \\
N\left(f^{-1} A \mid f^{-1} B\right) \leqslant N(A \mid B), & \text { (if } f \text { is surjective, } \\
N\left(f^{-1} Y, f^{-1} A\right) \leqslant N(Y, A), & \text { then the equalities hold) }
\end{aligned}
$$

$$
\begin{gathered}
N(A \vee B \mid O) \leqslant N(A \mid O) \cdot N(B \mid A \vee C), \\
N(A \vee B \mid O \vee D) \leqslant N(A \mid O) \cdot N(B \mid D), \\
N(Y, A \vee B) \leqslant N(X, A) \cdot N(Y, B), \\
N(A) \leqslant N(B) \cdot N(A \mid B),
\end{gathered}
$$$$
N(A \mid B) \leqslant N(A \mid O) \cdot N(C \mid B) \text {. }
$$

Inequalities (1.2)-(1.5) are obvious. For the proof of (1.6) let us fix $e \in O$. Then there exists $E \subset A$ such that $c \subset \cup E$ and $\operatorname{Card} E \leqslant N(A \mid C)$.
Now for any $a \in E$ there exists $B_{a} \subset B$ such that $c \cap a \subset \cup B_{a}$ and Card $B_{a}$ $\leqslant N(B \mid A \vee C)$. The family $\left\{a \cap b: a \in E, b \in B_{a}\right\}$ is a subfamily of $A \vee B$, its cardinality is not greater than $N(A \mid C) \cdot N(B \mid A \vee C)$ and its union contains $c$.

Inequalities (1.7)-(1.10) can easily be obtained from (1.6) by the use of (1.1) and (1.3).

In view of (1.4) and (1.7) the sequence $\left(\log N\left(A^{n} \mid B^{n}\right)\right)_{n=1}^{\infty}$ is subadditive; therefore there exists a limit

$$
\text { " } \quad \lim _{n \rightarrow \infty} \frac{1}{n} \log N\left(A^{n} \mid B^{n}\right)=h(f, A \mid B),
$$

which will be called the conditional entropy of $f$ on the cover $A$ with respect to the cover $B$. Moreover, we have

$$
\text { (1.11.) } \quad h(f, A \mid B) \leqslant \log N \cdot(A \mid B) \text {. }
$$

Notice that if $A$ is an open cover, then $h(f, A \mid \Theta)$ is equal to the familiar topological entropy of $f$ on the cover $A h(f, A)$.

Further, let

$$
h(f, Y, A)=\limsup _{n \rightarrow \infty} \frac{1}{n} \log N\left(Y, A^{n}\right)
$$

for $X \subset X, A \in \mathscr{P}$. This number is, in general, different from the analogous ones appearing in [4], [6].

Of course, $h(f, X, A)=h(f, A)$. From (1.2)-(1.10). we obtain the following inequalities (we list only those which will be necessary in the sequel):

(1.1.2) $\quad h(f, Y, A) \leqslant h(f, Z, B)$ for $B \geqslant A, Y \subset Z$,

(1.13) $\quad h(f, A \mid B) \leqslant h(f, C \mid D)$ for $C \geqslant A, B \geqslant D$,

(1.1.4) $\quad h\left(f, f^{-1} A \mid f^{-1} B\right) \leqslant h(f, A \mid B), \quad$ (if $f$ is surjective,

(1.15) $\left.\quad h\left(f, f^{-1} Y, f^{-1} A\right) \leqslant h(f, Y, A),\right\}$ then the equalities hold)

$$
\begin{gathered}
h(f, A) \leqslant h(f, B)+h(f, A \mid B), \\
h(f, A \mid B) \leqslant h(f, A \mid O)+h(f, C \mid B) .
\end{gathered}
$$

In view of (1.13) there exists a limit (finite or infinite)

$$
\operatorname{limix}_{\mathcal{A} \in \mathbb{L}} h(f, A \mid B)=\sup _{\mathcal{A} \in \mathbb{Q}} h(f, A \mid B)=h(f \mid B) \text {. }
$$

Wo call $h(f \mid B)$ the conditional entropy of $f$ with respect to the cover $B$. Notice that $h(f)=h(f \mid \Theta)$ is the topological entropy of $f$.

In view of (1.12) there also exists a limit

$$
\lim _{A, \mathcal{N C}} h(f, Y, A)=\sup _{A \in 2 T} h(f, Y, A)=h(f, Y) .
$$

Of: comse, $h(f, X)=h(f)$. 
From (1.12)-(1.17) we obtain:

$$
\begin{gathered}
h(f, Y) \leqslant h(f, Z) \quad \text { for } \quad Y \subset Z, \\
h(f \mid A) \leqslant h(f \mid B) \text { for } A \geqslant B, \\
h\left(f \mid f^{-1} B\right)=h(f \mid B), \\
\left.h\left(f, f^{-1} Y\right)=h(f, Y),\right\} \text { if } f \text { is a homeomorphism } \\
h(f) \leqslant h(f, B)+h(f \mid B) .
\end{gathered}
$$

To prove (1.20) and (1.21) it should be noted that $\left\{f^{-1} A: A \in \mathfrak{U}\right\}=\mathfrak{A}$ if $f$ is a homeomorphism.

We have, from the definition,

$$
h(f, A \mid B) \leqslant h(f \mid B) \quad \text { for } \quad A \in \mathfrak{A} .
$$

In view of (1.19) we can take the limit once more:

$$
\lim _{B \in \mathfrak{I}} h(f \mid B)=\inf _{B \in \mathfrak{U}} h(f \mid B)=h^{*}(f) .
$$

We call $h^{*}(f)$ the (topological) conditional entropy of the transformation $f$ (or, more precisely, of the cascade $(X, f)$ ).

From the definition we have

$$
\text { (1.24) } \quad h^{*}(f) \leqslant h(f \mid B) \text { for } B \in \mathfrak{A} \text {. }
$$

Putting $B=\Theta$, we obtain

$$
h^{*}(f) \leqslant h(f) .
$$

§ 2. Conneçtion with $h$-expansiveness. We first recall some notions from Bowen's paper [5]. Let $(X, d)$ be a compact metric space and $f$ : $X \rightarrow X$ a continuous transformation. The function $d_{n}$, defined by

$$
d_{n}(x, y)=\max _{0 \leqslant i \leqslant n-1} d\left(f^{i} x, f^{i} y\right),
$$

is a metric on $X$, equivalent to $d(n=1,2, \ldots)$. For a compact set $Y \subset X, \alpha>0, r_{n}(Y, \alpha)$ is the smallest cardinality of an $\alpha$-network for $Y$ in the metric $d_{n}$ (an $(n, a)$-spanning set for $Y$ ). Further, put

$$
\bar{r}(Y, \alpha)=\limsup _{n \rightarrow \infty} \frac{1}{n} \log r_{n}(Y, \alpha), \quad \tilde{h}(f, Y)=\lim _{\alpha \rightarrow 0} \bar{r}(Y, \alpha) .
$$

$B_{\alpha}^{n}(x)$ is a closed ball with the centre $x$ and radius $\alpha$ in the metric $a_{n}$,

Then

$$
\Phi_{a}(x)=\bigcap_{n=1}^{\infty} B_{\alpha}^{n}(x) \text {. }
$$

$$
h_{f}^{*}(\alpha)=\sup _{x \in \mathcal{X}} \tilde{h}\left(f, \Phi_{a}(x)\right)
$$

The transformation $f$ is called h-expansive if there exists an $\alpha>0$ such that

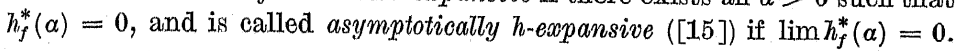

We shall point out the connection between these notions and the notions introduced in Section 1.

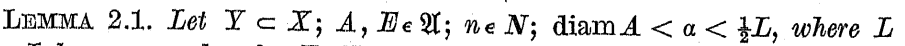
is the Lebesgue number for $\mathbb{E}$. Then

$$
N\left(Y, A^{n}\right) \leqslant r_{n}(Y, \alpha) \leqslant N\left(Y, B^{n}\right) .
$$

Proof. Let $e$ be the minimal $(n, \alpha)$-spanning set for $Y$. For $x \in e$, $k_{i}=0, \ldots, n-1$, we have $B_{\alpha}\left(f^{k} x\right) \prec E$; hence $B_{a}^{n}(x) \prec E^{n}$. But $\cup B_{a}^{n}(x)$ $\supset Y$, and so $r_{n}(X, \alpha) \leqslant N\left(Y, D^{n}\right)$. Let $\theta \subset A^{n}, \quad Y \subset \cup C$, Card $C$ $=N\left(X, A^{n}\right)$. Take one point from every $c \in C$ and let $b$ denote the set thus obtained. If $x \in c=\bigcap_{k=0}^{n-1} f^{-k} a_{k} \in C, a_{k} \in A$ for $k=0, \ldots, n-1$, then $a_{k} \subset B_{a}\left(f^{k} x\right)$ for $k=0, \ldots, n-1$; therefore, $c \subset B_{a}^{n}(x)$. Hence $b$ is an $(n, \alpha)$-spanning set and thus $N\left(Y, A^{n}\right) \leqslant r_{n}(Y, \alpha)$.

THEOREM 2.1. Let $X$ be a compact metric space, and let $(X, f)$ be a cascade, $\alpha>0, A, E \in \mathfrak{A}$, $\operatorname{diam} A<\alpha<\frac{1}{2} L$, where $L$ is the Lebesgue number for $\mathbb{t}$. Then.

$$
h(f \mid A) \leqslant h_{f}^{*}(\alpha)<h(f \mid E)
$$

Proof. Taking in Lemma 2.1 the limit with respect to $A, \mathbb{E} \in \mathfrak{X}$, $\alpha \rightarrow 0$, we obtain $\tilde{h}(f, Y)=h(f, Y)$ for a compact set $Y$. Thus, $h_{f}^{*}(\alpha)$ $=\sup _{x} h\left(f, \Phi_{\alpha}(\infty)\right)$. Let $O \in \mathfrak{U}$. The number $2 \alpha$ is smaller than the Lebesgue number of $E$; therefore (we use (1.2)): $N_{a}\left(\Phi_{\alpha}(x), O^{n}\right) \leqslant N\left(B_{\alpha}^{n}(x), C^{n}\right)$ $\leqslant N\left(C^{n} \mid E^{n}\right)$ for $n=1,2, \ldots$, and thus $h\left(f, \Phi_{\alpha}(x), C\right) \leqslant h(f, C \mid E)$. Talking the limit with respect to $O \in \mathfrak{A}$, we obtain $h\left(f, \Phi_{a}(x)\right) \leqslant h(f \mid E)$, and therefore $h_{f}^{*}(\alpha) \leqslant h(f \mid \mathbb{E})$.

Let $\beta>0, \delta>0$. Proposition 2.2 from [5] states that there exists a $\theta>0$ such that $r_{n}\left(B_{a}^{n}(x), \delta\right) \leqslant 0 \exp \left[\left(\beta+h_{f}^{*}(\alpha)\right) n\right]$ for $n=1,2, \ldots$, $\infty \in X$. Let $D \in \mathfrak{A}$ be a cover with the Lebesgue number greater than $2 \delta$. In view of Lemma 2.1, (1.2) and the inequality $\operatorname{diam} A<\alpha$, we have

$$
N\left(D^{n} \mid A^{n}\right) \leqslant \sup _{x \in \mathcal{X}} \mathcal{N}\left(B_{a}^{n}(x), D^{n}\right) \leqslant \sup _{x \in \mathbb{X}} r_{n}\left(B_{\alpha}^{n}(x), \delta\right) \leqslant c \exp \left[\left(\beta+h_{f}^{*}(\alpha)\right) n\right] .
$$

When $n \rightarrow \infty$, we obtain $h(f, D \mid A) \leqslant h_{f}^{*}(\alpha)+\beta$. Let $\delta \rightarrow 0, \beta \rightarrow 0$; we have $h(f, D \mid A) \leqslant h_{f}^{*}(\alpha)$ for every $D \in \mathfrak{A}$ and therefore $h(f \mid A) \leqslant h_{f}^{*}(\alpha)$.

CoRocicary 2.1. For a cascade $(X, f)$, where $X$ is a metric space,

(a) $f$ is h-expansive $\Leftrightarrow \exists h(f \mid A)=0$,

(b) $f$ is asymptotically $\stackrel{A \in \mathfrak{Q}}{h \text {-expansive }} \Leftrightarrow h^{*}(f)=0$. 
In the general case, where the space $X$ is not necessarily metrizable, the right-hand sides of (a) and (b) can serve as-definitions of $h$-expansiveness and asymptotical $h$-expansiveness, respectively.

\$ 3. Properties of conditional entropy. In this section we examine the basic properties of conditional entropy. The methods employed will enable us to simplify the proofs of some theorems concerning usual topological entropy.

In some proofs we shall use arguments of the theory of uniform structures (see Kelley [14]). For a compact Hausdorff space $X$, denote the set of all open symmetric neighbourhoods of the diagonal in $X \times X$ by $\mathfrak{N}(X)$ (simply $\mathfrak{N}$ if we consider only one space). It is the base for the uniform structure on $X$. The topology in $X$ defined by this structure coincides with the original one. For any open cover $A$ of $X$ there exists $L \in \mathfrak{R}$ such that $\{y:(x, y) \in L\} \prec A$ for any $x \in X$. Every such $L$ will be called a Lebesgue number for $A$.

Two important properties of conditional entropy have already been proved: they are (1.22) and (1.25). The next ones are:

Proposition 3.1. $h^{*}\left(f^{k}\right)=|k| \cdot h^{*}(f)$ for $k \in \boldsymbol{N}$; or for $k \in \boldsymbol{Z}$ if $f$ is a homeomorphism.

Proof. Observe that for a cover $A$ we have $\left(\dot{A}_{f}^{k}\right)_{f h}^{n}=A_{f}^{k n}$ and that for a fixed $k$ the family $\left\{A_{f}^{k}: A \in \mathfrak{U}\right\}$ is cofinal with $\mathfrak{2}$. This implies the proposition in the general case; in the case of a homeomorphism apply also (1.14).

Propostrion 3.2. Let $Y$ be a non-empty closed invariant subset of $X$ Then $h^{*}\left(\left.f\right|_{Y}\right) \leqslant h^{*}(f)$.

Proof. Let $A, B \in \mathfrak{A}(X)$. Then we have $N\left(\left(\left.A\right|_{Y}\right) \mid\left(\left.B\right|_{Y}\right)\right) \leqslant N(A \mid B)$ $\left(\left.A\right|_{Y}\right)^{n}=\left.A^{n}\right|_{\dot{Y}}$, and therefore $h\left(\left.f\right|_{Y},\left(\left.A\right|_{Y}\right) \mid\left(\left.B\right|_{Y}\right)\right) \leqslant h(f, A \mid B)$. To end the proof notice that $\left\{\left.A\right|_{X}: A \in \mathfrak{A}(X)\right\}=\mathfrak{U}(X)$.

From (1.22) immediately follows

Propostrron 3.3. If $h(f)=\infty$, then also $h^{*}(f)=\infty$.

PRoposinion 3.4. Let $X=Y_{1} \cup \ldots \cup Y_{n}$, where the sets $Y_{i}$ are nonempty, closed and invariant $(i=1, \ldots, r)$. Then $h^{*}(f)=\max h^{*}\left(\left.f\right|_{Y_{i}}\right)$.

Proof. The inequality $\max h^{*}\left(\left.f\right|_{Y_{i}}\right) \leqslant h^{*}(f)$ follows trom Proposition 3.2. Let $A, B \in \mathfrak{U}(X)$. For $n=1,2, \ldots, N\left(A^{n} \mid B^{n}\right) \leqslant \sum_{i=1}^{r} N\left(\left(\left.A^{n}\right|_{X_{i}}\right) \mid\left(\left.B^{n}\right|_{Y_{i}}\right)\right)$; hence

$$
h(f, A \mid B) \leqslant \max _{1 \leqslant i \leqslant r} h\left(\left(\left.f\right|_{Y_{i}}\right),\left(\left.A\right|_{Y_{i}}\right) \mid\left(\left.B\right|_{Y_{i}}\right)\right) \leqslant \max _{1 \leqslant i \leqslant r} h\left(\left(\left.f\right|_{Y_{i}}\right) \mid\left(\left.B\right|_{Y_{i}}\right)\right) .
$$

To end the proof notice that for any $B_{1}, \ldots, B_{r}$ such that $B_{i} \in \mathfrak{A}\left(Y_{i}\right)$, $i=1, \ldots, r$, there exists a $B \in \mathfrak{A}(X)$ such that $\left.B\right|_{r_{i}} \geqslant B_{i}$ for $i=1, \ldots, r$.
$A$ set $Y \subset X$ is called wandering if $f^{-n} Y \cap Y=\varnothing$ for $n=1,2, \ldots$ A point $x \in X$ is called non-wandering if it has no wandering open neighbourhood.

Let $\Omega$ denote the set of all non-wandering points for a transformation $f: X \rightarrow X$. Of course, $\Omega$ is non-empty, closed and invariant. We shall prove the following theorem, an analogue of Bowen's result concerning ustal topological entropy (cf. Bowen [3]):

Theorem 3.1. Let $(X, f)$ be a cascade, and let $\Omega$ be the set of non-wandering points for $f$. Then the conditional entropy of $f$ is attained on $\Omega$, i.e., $h^{*}(f)=h^{*}\left(\left.f\right|_{s}\right)$.

T.roof. The inequality $h^{*}\left(\left.f\right|_{\Omega}\right) \leqslant h^{*}(f)$ follows from Proposition 3.2 We shall prove the reverse inequality.

Let $B=\left\{b_{1}, \ldots, b_{r}\right\} \in \mathfrak{A}(\Omega)$. There exists a closed cover of the space $\Omega,\left\{q_{1}, \ldots, q_{r}\right\}$, wuch that $q_{i} \subset b_{i}$ for $i=1, \ldots, r$. The sets $q_{i}$ and $\Omega \backslash b_{i}$ are compact disjoint, and therefore there exists an open subset $c_{i}$ of a space $X$ such that $q_{i} \subset c_{i}$ and $\bar{c}_{i} \cap\left(\Omega \backslash b_{i}\right)=\varnothing$ (i.e. $\left.\bar{c}_{i} \cap \Omega \subset b_{i}\right) ; i=1, \ldots, r$. The set $X \backslash \bigcup_{i=1}^{r} c_{i}$ is compact and disjoint with $\Omega$. Hence there exists a finite family $H^{\prime}$ of wandering open subsets of $X$ such that $\cup F \supset X \backslash \bigcup_{i=1}^{r} c_{i}$. Therefore, $C=F \cup\left\{o_{1}, \ldots, c_{r}\right\}$ belongs to $\mathfrak{A}(X)$. Now let us take arbitrary $E \in \mathfrak{A}(X)$ and $\varepsilon>0$. Write $A=\left.E\right|_{\Omega} \in \mathfrak{A}(\Omega)$. Take $p$ such that

$$
\frac{1}{p} \log N\left(A^{p} \mid B^{p}\right) \leqslant h\left(\left(\left.f\right|_{\Omega}\right), A \mid B\right) \text {. }
$$

Jete $A=\left\{a_{1}, \ldots, a_{s}\right\}$; write

$$
\alpha=N\left(A^{p} \mid B^{p}\right) .
$$

The definition of $N\left(A^{p} \mid B^{p}\right)$ tells us that there exists a mapping $T$ from $\{1, \ldots, r\}^{p}$ (the Oartesian product of $p$ copies of the set $\{1, \ldots, r\}$ ) into the family of all subsets of the set $\{1, \ldots, s\}^{p}$, such that for any $\sigma \in\{1, \ldots, r\}^{p}$ :

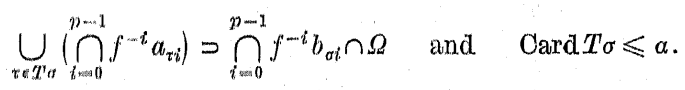

Let $d_{i}(i=1, \ldots, s)$ be an element of $E$ for which $a_{i}=d_{i} \cap \Omega$. For $\sigma \in\{1, \ldots, r\}^{x}$ we have

$$
\begin{aligned}
\left(\bigcap_{i=0}^{p-1} f^{-i} o_{\sigma i}\right) \cap \Omega & \left.=\prod_{i=0}^{p-1} f^{-i}\left(\overline{c_{\sigma i}} \cap \Omega\right)\right] \cap \Omega \subset\left(\bigcap_{i=0}^{p-1} f^{-i} b_{\sigma i}\right) \cap \Omega \\
& \subset \bigcup_{i \in T \sigma \sigma}\left(\bigcap_{i=0}^{p-1} f^{-i} a_{t i}\right) \subset \bigcup_{\tau \in T \sigma}\left(\bigcap_{i=0}^{p-1} f^{-i} d_{x i}\right)
\end{aligned}
$$


and thus

$$
\varnothing=\left[\bigcap_{i=0}^{p-1} f^{-i} \overline{c_{\alpha i}} \backslash \bigcup_{\tau \in T \sigma \sigma}\left(\bigcap_{i=0}^{p-1} f^{-i} d_{\tau i}\right)\right] \cap \Omega \supset \overline{\left[\bigcap_{i=0}^{p-1} f^{-i} c_{\sigma i} \backslash \bigcup_{\tau \in T}\left(\bigcap_{i=0}^{p-1} f^{-i} d_{\tau i}\right)\right]} \cap \Omega .
$$

Hence we obtain

$$
\bar{e} \cap \Omega=\varnothing
$$

where

$$
e=\bigcup_{\sigma \in\{1, \ldots, r\}^{p}}\left[\bigcap_{i=0}^{p-1} f^{-i} c_{\sigma i} \backslash \bigcup_{\tau \in T \sigma}\left(\bigcap_{i=0}^{p-1} f^{-i} d_{\tau i}\right)\right]
$$

Therefore there exists a finite family $G$ of open wandering subsets of $X$ such that

$$
\bigcup G \supset \bar{e} \cup\left(X \backslash \bigcup_{i=1}^{s} d_{i}\right)
$$

and $\left.G\right|_{\cup G} \geqslant\left. E\right|_{\cup G}$. Write $D=G \cup\left\{d_{1}, \ldots, d_{s}\right\}$. We have $D \in \mathfrak{A}(X), D \geqslant E$.

Now we fix $k \in \boldsymbol{N}$ and estimate the number $N\left(D^{k p p} \mid O^{k p p}\right)$ from above. Let $\varnothing \neq c \in C^{k p}$. Then $e=\bigcap_{j=0}^{k p-1} f^{-1} w_{j}$ for some sets $w_{j} \in C$. If a set $w$ belongs to $F$, then it occurs in the sequence $\left(w_{0}, \ldots, w_{k p-1}\right)$ at most once. Therefore we can divide the set $\{0, \ldots, k-1\}$ into two sets $P$ and $Q$ in such a way that $\operatorname{Card} P \leqslant \operatorname{Card} F$ and there exist elements $\sigma_{j} \epsilon\{1, \ldots, r\}^{p}$ for $j \in Q$ such that $c \subset \bigcap_{j=0}^{k-1} f^{-j p} v_{j}$, where

$$
v_{j}=\left\{\begin{array}{lll}
X & \text { for } & j \in P \\
\bigcap_{i=0}^{X-1} f^{-i} c_{\sigma_{j} i} & \text { for } & j \in Q .
\end{array}\right.
$$

In view of (3.4) and (3.5) we have

$$
\bigcap_{i=0}^{p-1} f^{-i} e_{\sigma i} \backslash \bigcup_{\tau \in T \sigma}\left(\bigcap_{i=0}^{p-1} f^{-i} d_{r i}\right) \subset e \subset \bigcup G
$$

for any $\sigma \in\{1, \ldots, r\}^{p}$, and thus for $j \in Q$

$$
v_{j} \subset \bigcup G \cup \bigcup_{\tau \in T \sigma_{j}}\left(\bigcap_{i=0}^{p-1} f^{-i} d_{\tau i}\right)=\bigcup\left(G \vee f^{-1} D^{p-1}\right) \cup \bigcup_{\tau \in T \sigma_{j}}\left(\bigcap_{i=0}^{p-1} f^{-i} d_{v i}\right) .
$$

For $j \in P$ we have $v_{j}=X \subset \cup D^{p}$. Finally

(3.6) $\quad c \subset \bigcap_{j \in Q} f^{-j p}\left[\bigcup\left(G \vee f^{-1} D^{p-1}\right) \cup \bigcup_{\tau \in T \sigma_{j}}\left(\bigcap_{i=0}^{p-1} f^{-i} d_{\tau i}\right)\right] \cap \bigcap_{j \in P} f^{-j p}\left(\bigcup D^{p}\right)$.
The right-hand side of (3.6) can be rewritten as the union of a certain family $H \subset D^{k n}$. If $\varnothing \neq d \in H, d=\bigcap_{i=0}^{k p-1} f^{-i} u_{i}, u_{i} \in D$, then $\operatorname{Card}\left\{i: u_{i} \in G\right\}$ $\leqslant$ Card $G$. Therefore from (3.6) we obtain

$$
\begin{aligned}
& \text { Oard }(H \backslash\{\varnothing\}) \\
& \quad \leqslant \sum_{n=0}^{\operatorname{Card} G}\left[\left(\begin{array}{c}
\operatorname{Card} Q \\
n
\end{array}\right) \cdot\left(\operatorname{Card} G \cdot \operatorname{Card}\left(D^{p-1}\right)\right)^{n} \cdot \alpha^{\operatorname{Card} Q-n} \cdot\left(\operatorname{Card}\left(D^{p}\right)\right)^{\operatorname{Card} P}\right] \\
& \quad \leqslant(\operatorname{Oard} G+1) \cdot k^{\operatorname{Card} Q} \cdot\left(\operatorname{Oard} G \cdot \operatorname{Card}\left(D^{p-1}\right)\right)^{\operatorname{Card} G} \cdot \alpha^{k} \cdot\left(\operatorname{Card}\left(D^{p}\right)\right)^{\operatorname{Card} F}
\end{aligned}
$$

Hence we have $N\left(D^{k p p} \mid O^{k p p}\right) \leqslant \beta \cdot k^{p} \cdot \alpha^{k}+1$ for some constants $\beta$ and $\gamma$ independent of $k$ and thus

$$
h(f, D \mid C) \leqslant \frac{1}{p} \log \alpha .
$$

But $D \geqslant F$, and therefore (3.1), (3.2), (3.7), (1.13) and (1.23) imply

$$
h(f, E \mid O) \leqslant h\left(\left(\left.f\right|_{\Omega}\right), A \mid B\right)+\varepsilon \leqslant h\left(\left(\left.f\right|_{\Omega}\right) \mid B\right)+\varepsilon .
$$

We have thus obtained the following result:

$$
\underset{B \in \mathbb{2 r}(\Omega)}{\forall} \underset{O \in \operatorname{dr}(X)}{\exists} \underset{\substack{H \in \in \\ B>0}}{\forall} h(X) .
$$

Taking the limit with respect to $E \in \mathfrak{A}(X)$ and $\varepsilon \rightarrow 0$, we obtain

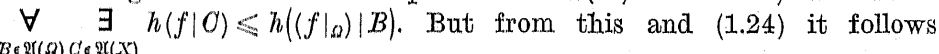
that $h^{*}(f) \leqslant h^{*}\left(\left.f\right|_{\Omega}\right)$.

In the proofs of the further properties we shall have to be able to refine a given open cover in an essential way. For this purpose define

(a) the star of a set $Y$ with respect to a cover $A$

$$
\operatorname{st}(Y, A)=\bigcup\{a \in A: a \cap Y \neq \varnothing\},
$$

(b) the star of a cover $A$

$$
\operatorname{St} A=\{\operatorname{st}(a, A): a \epsilon A\}
$$

(notice that the above definition is different from the one commonly ased).

Ot' course, for $A \in \mathfrak{A}$ we have St $A \in \mathfrak{A}$; also $A \geqslant \operatorname{St} A$.

Proposriton 3.5. The family $\{\mathrm{St} A: A \in \mathfrak{X}\}$ is cofinal with $\mathfrak{A}$.

Proof. Tet $A \in \mathfrak{A}$ and let $T_{\in} \mathfrak{R}$ be a Lebesgue number for $A$. There existis a $U \in \mathfrak{N}$ such that $U \circ U \circ U \subset L$ (where $U \circ V=\{(x, y) \in X \times X$ : $\exists(x, \approx) \in U,(\approx, \infty) \in V\})$. Then any finite cover chosen from the open cover $\{\{y \in X:(x, y) \in U\}\}_{\infty \in X}$ is a refinement of $A$. 
A family $A$ of subsets of a space $X$ will be called a discover if elements of $A$ are pairwise disjoint.

The application of the notion of stars is based on the following three lemmas :

Lemma 3.1. Let $A$ be a finite oove of $X, Y \subset X$. Then there exists a discover $B \subset A$ such that

$$
b \cap Y \neq \varnothing \quad \text { for } \quad b \in B,
$$

$$
Y \subset \bigcup\{\operatorname{st}(b, A): b \in B\} \text {. }
$$

Proof. Assume that $B^{\prime} \subset A$ is a discover fulfilling (a) but not (b). Then there exists a point $y \in Y$ which does not belong to the set $\bigcup\{$ st $(b, A)$ : $\left.b \in B^{\prime}\right\}$. Take $a \epsilon A$ such that $y \in a$. Then $B^{\prime \prime}=B^{\prime} \cup\{a\}$ is also a discover contained in $A$ and fulfilling (a). Now the lemma follows from the finiteness of $A$ (notice that $\varnothing$ is a discover fulfilling (a)).

LEMma 3.2. Let $A$ be a finite cover of $X ; Y \subset X ;$ let $B$ be a discovor contained in st $A$ such that $b \cap Y \neq \emptyset$ for $b \in B ; C \subset A$, st $(Y, A) \subset \bigcup C$. Then Card $C \geqslant$ Card $B$.

Proof. Take $a \in A$ such that $s t(a, A) \in B$. Then $a \cap s t(Y, A) \neq \varnothing$, and thus there exists a $c_{a} \in O$ such that $a \cap c_{a} \neq \varnothing$. But then $c_{a} \subset \operatorname{st}(a, A) \in B$. $B$ is a discover and therefore $c_{a} \neq c_{d}$ provided $\operatorname{St}(a, A) \neq \operatorname{St}(d, A)$. Thus Card $C \geqslant \operatorname{Card} B$.

Lemma 3.3. Let $A$ be a cover. Then $\operatorname{St}\left(A^{k}\right) \geqslant(\mathrm{St} A)^{k}$ for $k=1,2, \ldots$

Proof. If $a_{i} \in A$ for $i=0, \ldots, k-1$, then

$$
\text { sti }\left(\bigcap_{i=0}^{k-1} f^{-i} a_{i}, A^{k}\right) \subset \operatorname{st}\left(f^{-j} a_{j}, f^{-j} A\right)=f^{-j}\left(\operatorname{st}\left(a_{j}, A\right)\right)
$$

for $j=0, \ldots, k-1$; therefore

Thus $\operatorname{St}\left(A^{k}\right) \geqslant(\text { St } A)^{k}$.

$$
\operatorname{st}\left(\bigcap_{i=0}^{k-1} f^{-i} a_{i}, A^{k}\right) \subset \bigcap_{j=0}^{k-1} f^{\cdots j} \operatorname{st}\left(a_{j}, A\right) .
$$

For cascades $\left(X_{i}, f_{i}\right), i \in I$, denote by $\prod_{i \in I} f_{i}:\left.\prod_{i \in I} X_{i} \rightarrow\right|_{i \in I} X_{i}\left(f_{1} \times \ldots \times f_{n}\right.$ in a finite case) the transformation given by the formula $\prod_{i \in I} f_{i}\left(x_{i}\right)_{i e t}$ $=\left(f_{i} x_{i}\right)_{i \epsilon,}$. For families $A_{i}$ of subsets of spaces $X_{i}(i=1,2)$, respectively, write $A_{1} \times A_{2}=\left\{a_{1} \times a_{2}: a_{i} \in A_{i}, i=1,2\right\}$. The operations st and St, as can easily be seen, commute with the operation of taking a product.

LEMMA 3.4. Let $\left(X_{1}, f_{1}\right),\left(X_{2}, f_{2}\right)$ be cascades, $\varnothing \neq Y_{i} \subset X_{i}$ and let $A_{i}$ be a finite cover of $X_{i}(i=1,2)$. Then

(a) $N\left(Y_{1}, \operatorname{StSt} A_{1}\right) \cdot N\left(Y_{2}, \operatorname{StSt} A_{2}\right) \leqslant N\left(\operatorname{st}\left(Y_{1} \times Y_{2}, A_{1} \times A_{2}\right), A_{1} \times A_{2}\right)$,

$$
N\left(Y_{1} \times Y_{2}, A_{1} \times A_{2}\right) \leqslant N\left(Y_{1}, A_{1}\right) \cdot N\left(Y_{2}, A_{2}\right)
$$

Troof. The inequality (b) is obvious. We shall prove (a).

In view of Lemma 3.1. there exist discovers $B_{i} \subset$ St $A_{i}$ such that $b \cap Y_{i} \neq \varnothing$ for $b \in B_{i}$ and $Y_{i} \subset \bigcup\left\{\operatorname{st}\left(b, \operatorname{St} A_{i}\right): b \in B_{i}\right\} \quad(i=1,2)$. The last inclusion implies ( $\operatorname{ard} B_{i} \geqslant N\left(Y_{i}\right.$, StSt $\left.A_{i}\right)$. We have $B_{1} \times B_{2}$ $\subset \mathrm{St}, A_{1} \times S \mathrm{t} . A_{2}=\operatorname{St}\left(A_{1} \times A_{2}\right) . B_{1} \times B_{2}$ is a discover; moreover $b \cap\left(Y_{1} \times Y_{2}\right)$ $\neq \varnothing$ lor $b \in B_{1} \times B_{2}$, and therefore, in view of Lemma 3.2 , if $\bigcup C \supset$ st $\left(Y_{1} \times Y_{2}, A_{1} \times A_{2}\right)$ for some $O \subset A_{1} \times A_{2}$, then $\operatorname{Card} C \geqslant \operatorname{Card}\left(B_{1} \times B_{2}\right)$ $=\operatorname{Oard} B_{1} \cdot$ Oard $B_{2}$.

Now wo are able to prove the next property of conditional entropy:

Treorkim 3.2. Let $\left(X_{1}, f_{1}\right),\left(X_{2}, f_{2}\right)$ be cascades. Then

$$
h^{*}\left(f_{1} \times f_{2}\right)=h^{*}\left(f_{1}\right)+h^{*}\left(f_{2}\right) \text {. }
$$

Proot. From Lenma 3.4 (b) it follows that

$$
h\left(f_{1}, A_{1} \mid B_{1}\right)+h\left(f_{2}, A_{2} \mid B_{2}\right) \geqslant h\left(f_{1} \times f_{2}, A_{1} \times A_{2} \mid B_{1} \times B_{2}\right)
$$

for $A_{i}, B_{i} \in \mathfrak{A}\left(X_{i}\right), i=1,2$. In view of the fact that $\left\{A_{1} \times A_{2}: A_{i} \in \mathfrak{A}\left(X_{i}\right)\right.$, $i=1,2\}$ is cotinal with $\mathfrak{A}\left(X_{1} \times X_{2}\right)$, this inequality yields $h^{*}\left(f_{1} \times f_{2}\right)$ $\leqslant h^{*}\left(f_{1}\right)+h^{*}\left(f_{2}\right)$. Let $A_{i}, B_{i} \in \mathfrak{Q}\left(X_{i}\right), A_{i} \geqslant B_{i}$ for $i=1,2$. We have

$$
\operatorname{st}\left(b_{1} \times b_{2}, A_{1} \times A_{2}\right) \subset \operatorname{st}\left(b_{1} \times b_{2}, B_{1} \times B_{2}\right) \in \operatorname{St}\left(B_{1} \times B_{2}\right)
$$

for $b_{i} \in B_{i}, i \ldots$ 1. 2. Lemma 3,4 (a) implies for $n=1,2, \ldots$

$$
N\left(\operatorname{St}_{1} \operatorname{St}_{1}\left(A_{1}^{n}\right) \mid B_{1}^{n}\right) \cdot N\left(\operatorname{StSt}\left(A_{2}^{n}\right) \mid B_{2}^{n}\right) \leqslant N\left(\left(A_{1} \times A_{2}\right)^{n} \mid \operatorname{St}\left(\left(B_{1} \times B_{2}\right)^{n}\right)\right) .
$$

Hence, in view of Temma 3.3 and (1.3):

$$
h\left(f_{1}, \operatorname{stst} A_{1} \mid B_{1}\right)+h\left(f_{2}, \text { stst } A_{2} \mid B_{2}\right) \leqslant h\left(f_{1} \times f_{2}, A_{1} \times A_{2} \mid \operatorname{St}\left(B_{1} \times B_{2}\right)\right) .
$$

In view of Proposition ' 3.5 and the fact that the family $\left\{A_{1} \times A_{2}\right.$ : $\left.A_{i \in \mathfrak{A}}\left(X_{i}\right), i=\mathbf{1}, 2\right\}$ is cofinal with $\mathfrak{A}\left(X_{1} \times X_{2}\right)$, the above inequality gives the inequalitity $h^{*}\left(f_{1}\right)+h^{*}\left(f_{2}\right) \leqslant h^{*}\left(f_{1} \times f_{2}\right)$.

Remark 3.1. Theorem 3.2 can be generalized by induction to the product of an whitrary finitie number of transformations: $h^{*}\left(f_{1} \times \ldots \times f_{n}\right)$ $=\sum_{i=1}^{n} h_{i}^{*}\left(f_{i}\right)$.

Romark 3.2. T'utting in the proof of Theorem $3.2 B_{i}=\Theta\left(X_{i}\right)$, we obtain a simple proof of the theorem on the topological entropy of the product due to (xoodwyn [1.1]: $h\left(f_{1} \times f_{2}\right)=h\left(f_{1}\right)+h\left(f_{2}\right)$. This theorem can, in the same way, be generalized by induction to the product of an arbitrary finite number of transformations. The foregoing considerations result finally in anow joot of the following theorem (also due to Goodwyn [11]): 
THEOREM 3.3. Let $\left(X_{i}, f_{i}\right)$ be cascades $(i=1,2, \ldots)$. Then

$$
h\left(\prod_{i=1}^{\infty} f_{i}\right)=\sum_{i=1}^{\infty} h\left(f_{i}\right)
$$

Proof. Write

$\mathfrak{A}_{k}=\left\{A \times\left\{\prod_{i=k}^{\infty} X_{i}\right\}: A \in \mathfrak{A}\left(\prod_{i=1}^{k-1} X_{i}\right)\right\} \quad$ for $k=1,2, \ldots ; \quad \mathfrak{A}_{0}=\mathfrak{A}\left(\prod_{i=1}^{\infty} X_{i}\right)$.

The family of open sets $\left\{a \times \prod_{i=1 k}^{\infty} X_{i}: a\right.$ is an open subset of $\prod_{i=1}^{k-1} X_{i}$, $k=1,2, \ldots\}$ is a base of the space $\prod_{i=1}^{\infty} X_{i}$, and therefore the family $\bigcup_{k=1}^{\infty} \mathfrak{A}_{k}$ is cofinal with $\mathfrak{A}_{0}$. Denote by $\pi_{k}: \prod_{i=1}^{\infty} X_{i} \rightarrow \prod_{i=1}^{l-1} X_{i}$ the natural projection. Notice that the sequence $\left(\mathfrak{U}_{n}\right)_{n=1}^{\infty}$ is ascending and that $h\left(\prod_{i=1}^{\infty} f_{i}, A\right)$ $=h\left(\prod_{i=1}^{k-1} f_{i}, \pi_{k} A\right)$ for $A \in \mathfrak{A}_{k}$. Hence

$$
\begin{aligned}
h\left(\prod_{i=1}^{\infty} f_{i}\right) & =\lim _{k \rightarrow \infty} \lim _{A \in \mathbb{N}} h\left(\prod_{i=1}^{\infty} f_{i}, A\right)=\lim _{k \rightarrow \infty} h\left(\prod_{i=1}^{k-1} f_{i}\right) \\
& =\lim _{k \rightarrow \infty} \sum_{i=1}^{k-1} h\left(f_{i}\right)=\sum_{i=1}^{\infty} h\left(f_{i}\right) .
\end{aligned}
$$

Concerning conditional entropy, the following analogouss, theorem also holds:

TheOREM 3.4. Let $\left(X_{i}, f_{i}\right)$ be cascades $(i=1,2, \ldots), h\left(\prod_{i=1}^{\infty} f_{i}\right)<\infty$.

$$
h^{*}\left(\prod_{i=1}^{\infty} f_{i}\right)=\sum_{i=1}^{\infty} h^{*}\left(f_{i}\right) .
$$

Proof. We have

$$
h^{*}\left(\prod_{i=1}^{\infty} f_{i}\right)=h^{*}\left(\prod_{i=1}^{k-1} f_{i}\right)+h^{*}\left(\prod_{i=1 / t_{i}}^{\infty} f_{i}\right)=\sum_{i=1}^{k-1} h^{*}\left(f_{i}\right)+h^{*}\left(\prod_{i=1 / l_{c}}^{\infty} f_{i}\right)
$$

But $0 \leqslant h^{*}\left(\prod_{i=k}^{\infty} f_{i}\right) \leqslant h\left(\prod_{i=k_{i}}^{\infty} f_{i}\right)=\sum_{i=k_{i}}^{\infty} h\left(f_{i}\right)$ for $k=1,2, \ldots$, and therefore $\lim _{k \rightarrow \infty} h^{*}\left(\prod_{i=k}^{\infty} f_{i}\right)=0$. Thus

$$
h^{*}\left(\prod_{i=1}^{\infty} f_{i}\right)=\lim _{k \rightarrow \infty} \sum_{i=1}^{k-1} h^{*}\left(f_{i}\right)=\sum_{i=1}^{\infty} h^{*}\left(f_{i}\right)
$$

\$ 4. Connection with measure-theoretic entropy. Now we shall examine the connection between topological conditional entropy and measuretheoretic entropy.

Denote by $\mathfrak{M}(X)$ the space of all Borel regular normed measures on $X$ and by $\mathfrak{M}(X, f)$ the subspace of those measures from $\mathfrak{M}(X)$ which are invariant with respect to $f$. We shall consider these spaces with the weak." topology. It is a well-known fact that they are both compact.

Now we prove an important fact, which allows us to simplify some prools (e.g. Bowen [5], [6], Goodwyn [10]) and to give a very short proof of Goodwyn's theorem. This fact can be found in Denker's paper [7] though it is not explicitly formulated there.

Propostrion 4.1. Let $\mu \in \mathfrak{M}(X, f)$; let $A$ be a finite Borel partition of $X$. Then there exist a finite Borel partition $B$ of $X$ and a cover $C \epsilon \mathfrak{A}(X)$ such that

$$
\begin{gathered}
h_{\mu}(f, B) \geqslant h_{\mu}(f, A)-1, \\
N(B \mid O) \leqslant 2 .
\end{gathered}
$$

Proof. Let $A=\left\{a_{1}, \ldots, a_{r}\right\}$. There exists a number $\varepsilon>0$ such that if $\mu\left(a_{i}-b_{i}\right) \leqslant \varepsilon$ for $i=1, \ldots, r$ for a partition $B=\left\{b_{0}, b_{1}, \ldots, b_{r}\right\}$, then the condition (a) holds (see e.g. Smorodinsky [16], Lemma 5.8). We choose compact sets $b_{i}$ contained in $a_{i}$ such that $\mu\left(a_{i} \backslash b_{i}\right) \leqslant \varepsilon$ for $i=1, \ldots, r$ (this is possible because the measure $\mu$ is regular) and we take $b_{0}=X \backslash \bigcup_{i=1}^{r} b_{i}$. Now we define $O=\left\{c_{1}, \ldots, c_{r}\right\}, c_{i}=b_{0} \cup b_{i}$ for $i=1, \ldots, r . O_{\in} \in \mathfrak{A}$ and (b) also holds.

Trroorem 4.1 (Goodwyn [10]). Let $(X, f)$ be a cascade, $\mu \in \mathfrak{M}(X, f)$. Then $h_{\mu}(f) \leqslant h(f)$.

Proof. Let $A$ be a finite Borel partition of $X, B$ and $C$ as in Proposition 4.1. The well-known inequality $H_{\mu}\left(B^{n}\right) \leqslant \log N\left(B^{n}\right)$ for $n=1,2, \ldots$ gives $h_{\mu}(f, B) \leqslant h(f, B)$. In view of Proposition 4.1, (1.11). and (1.16) we get

$$
\begin{aligned}
h_{\mu}(f, A) \leqslant h_{\mu}(f, B)+1 \leqslant h(f, B)+1 & \leqslant h(f, C)+h(f, B \mid C)+1 \\
& \leqslant h(f)+(\log 2+1) .
\end{aligned}
$$

$A$ has been arbitrary, and therefore $h_{\mu}(f) \leqslant h(f)+(\log 2+1)$. But this is true for every continuous transformation of $X$ into itself for which $\mu$ is invariant, in particular for $f^{n}$ instead of $f$. Hence, for $n=1,2, \ldots$,

$$
h_{\mu}(f)=\frac{1}{n} h_{\mu}\left(f^{n}\right) \leqslant \frac{1}{n} h\left(f^{n}\right)+\frac{1}{n}(\log 2+1)=h(f)+\frac{1}{n}(\log 2+1) .
$$

Therefore, $h_{\mu}(f) \leqslant h(f)$. 
In the sequel we shall need the following simple inequality:

Lemma 4.1. For finite Borel partitions $A$ and $B$,

$$
H_{\mu}(A \mid B) \leqslant \log N(A \mid B) .
$$

Proof. For any $b \in \cdot B$

and therefore

$$
H_{\left.\mu\right|_{b} / \mu(b)}\left(\left.A\right|_{b}\right) \leqslant \log N(b, A) \leqslant \log N(A \mid B),
$$

$$
H_{\mu}(A \mid B)=\sum_{b \in B} \mu(b) \cdot H_{\left.\mu\right|_{b} / \mu(b)}\left(\left.A\right|_{b}\right) \leqslant \log N(A \mid B) .
$$

Formula (1.22) is valid also for measure-theoretic entropy, namely:

Proposition 4.2 (cf. Bowen [5]). Let $D$ be a finite Borel partition of $X, \mu \in \mathfrak{M}(X, f)$. Then

$$
h_{\mu}(f) \leqslant h_{\mu}(f, D)+h(f \mid D) .
$$

Proof. Let $A$ be a finite Borel partition of $X$ and let $B$ and $C$ be as in Proposition 4.1. In view of. Lemma 4.1,

$$
H_{\mu}\left(B^{n}\right) \leqslant H_{\mu}\left(D^{n}\right)+H_{\mu}\left(B^{n} \mid D^{n}\right) \leqslant H_{\mu}\left(D^{n}\right)+\log N\left(B^{n} \mid D^{n}\right)
$$

$$
\text { for } n=1,2, \ldots \text {, }
$$

and therefore $h_{\mu}(f, B) \leqslant h_{\mu}(f, D)+h(f, B \mid D)$. Applying Proposition 4.1, (1.11) and (1.17), we obtain

$$
\begin{aligned}
h_{\mu}(f, A) & \leqslant h_{\mu}(f, B)+1 \leqslant h_{\mu}(f, D)+h_{h}(f, B \mid D)+1 . \\
& \leqslant h_{\mu}(f, D)+h(f, O \mid D)+h(f, B \mid C)+1 \\
& \leqslant h_{\mu}(f, D)+h(f \mid D)+(\log 2+1) .
\end{aligned}
$$

$A$ has been arbitrary, and therefore $h_{\mu}(f) \leqslant h_{\mu}(f, D)+h(f \mid D)+(\log 2+1)$. But this is true for every continuous transformation of $X$ into itself for which $\mu$ is invariant and for every finite Borel partition of $X$, and therefore for $n=1,2, \ldots$ (it is easy to see that $h_{\mu}\left(f^{n}, D_{f}^{n}\right)=n h_{\mu}(f, D)$ and $\left.h\left(f^{n} \mid D_{f}^{n}\right)=n h(f \mid D)\right)$ :

$$
\begin{aligned}
h_{\mu}(f) & =\frac{1}{n} h_{\mu}\left(f^{n}\right) \leqslant \frac{1}{n} h_{\mu}\left(f^{n}, D_{f}^{n}\right)+\frac{1}{n} h\left(f^{n} \mid I_{f}^{n}\right)+\frac{1}{n}(\log 2+1) \\
& =h_{\mu}(f, D)+h(f \mid D)+\frac{1}{n}(\log 2+1)
\end{aligned}
$$

Thuis $h_{\mu}(f) \leqslant h_{\mu}(f, D)+h(f \mid D)$.

Let us consider the measure-theoretic entropy regarded as a function of a measure: $h_{.}(f): \mathfrak{M}(X, f) \rightarrow \overline{\boldsymbol{R}}_{\zeta}$ Denote by $h_{\mu}^{*}(f)=\limsup _{p \rightarrow \mu} h_{\nu}(f)-h_{\mu}(f)$ (we assume $\infty-\infty=0$ ) for $\mu \in \mathfrak{M}(X, f)$. Notice that $h_{l}^{*}(f)=0$ iff $h_{.}(f)$ is upper semicontinuous at the point $\mu$.
Now we generalize Theorem 3 of [1.5].

Tirworman 4.2. Let $(X, f)$ be a cascade, $\mu \in \mathfrak{M}(X, f)$. Then $h_{\mu}^{*}(f) \leqslant h^{*}(f)$. Proof. If $h(f)=\infty$, then, in view of Proposition 3.3, also $h^{*}(f)=\infty$. Hence we can assume $h(f)<\infty$. Let $A=\left\{a_{1}, \ldots, a_{r}\right\} \in \mathfrak{A}$. Take a cover $B=\left\{b_{1}, \ldots, b_{r}\right\} \in \mathfrak{A}$ such that $\overrightarrow{b_{i}} \subset a_{i}$ for $i=1, \ldots, r$. Then take continuous functions $p_{i}: X \rightarrow[0,1]$ such that $p_{i} x=1$ for $x \in \overline{b_{i}}$ and $p_{i} x=0$ for $x_{\phi} a_{i}, i=1, \ldots, r$. For some $a \in[0,1]$ we have $\mu\left(\bigcup_{i=1}^{r} p_{i}^{-1}\{\alpha\}\right)=0$; therefore the finite Borel partition $C=\left\{\varphi_{1}^{-1}[\alpha, 1], \varphi_{2}^{-1}[\alpha, 1] \backslash p_{1}^{-1}[\alpha, 1], \ldots\right.$ $\left.\ldots, p_{r}^{-1}[\alpha, 1] \backslash \bigcup_{i=1}^{r-1} p_{i}^{-1}[\alpha, 1]\right\}$ consists of sets with boundaries of measure 0 . We also have $O \geqslant A$. Take $\varepsilon>0$. For a fixed $n$, the partition $O^{n}$ consists also of sets with boundaries of measure 0 , and therefore for some open neighbourhood. $U_{n} \subset \mathfrak{M}(X, f)$ of $\mu$ we have $\frac{1}{n} H_{\mu}\left(C^{n}\right) \geqslant \frac{1}{n} H_{p}\left(C^{n}\right)-\varepsilon$ whenever $\nu \in U_{n}$. Thus, in view of Proposition 4.2,

$$
\begin{aligned}
h_{\mu}(f) \geqslant h_{\mu}(f, O) & =\lim _{n \rightarrow \infty} \frac{1}{n} H_{\mu}\left(C^{n}\right) \geqslant \operatorname{limsupsup}_{n \rightarrow \infty} \frac{1}{\nu \in U_{n}} \frac{1}{n} H_{\nu}\left(C^{n}\right)-\varepsilon \\
& \geqslant \limsup _{n \rightarrow \infty} \sup _{\nu \in U_{n}} h_{\nu}(f, O)-\varepsilon \geqslant \limsup _{n \rightarrow \infty} \sup _{\nu \in U_{n}} h_{\nu}(f)-h(f \mid O)-\varepsilon \\
& \geqslant \limsup _{\nu \rightarrow \mu} h_{\nu}(f)-h(f \mid A)-\varepsilon .
\end{aligned}
$$

$\varepsilon$ has been arbitrary, and therefore $h_{\mu}^{*}(f) \leqslant h(f \mid A)$. But $A$ has also been arbitrary, and thus $h_{\mu}^{*}(f) \leqslant h^{*}(f)$.

OOROLTARY 4.1. (cf. [15]). If $f$ is asymptotically h-expansive, then there exists a measure with maximal entropy for $f$.

Proof. If $f$ is asymptotically $h$-expansive, then $h(f)$ is an upper semicontinuous function on the compact space $\mathfrak{M}(X, f)$, and therefore it attains its supremum.

Remark 4.1. Dinaburg's theorem ([8], proof in the general case $[9],[1.7])$ assertis that the topological entropy of a transformation $f$ is the best, estimation of the measure-theoretic entropies of $f$, i.e., $h(f)$ $=\sup _{\mu} h_{\mu}(f)$. But an analogous theorem for $h^{*}$ and $h_{\mu}^{*}$ is not valid (see $\mu \in \mathbb{M}(X, f)$

In some cases the following fact will be useful for the computation of topological conditional entropy:

PROPOSTIION 4.3. Let $\left(Y_{n}\right)_{n=1}^{\infty}$ be a descending sequence of invariant olosed subsets of $X$. Let $\left(U_{n}\right)_{n=1}^{\infty}$ be a descending sequence of open subsets 

of $X$ such that $Y_{n} \subset U_{n}$ for $n=1,2, \ldots$ and $\bigcap_{n=1}^{\infty} U_{n}=\bigcap_{n=1}^{\infty} Y_{n}=Y \neq \varnothing$.
Then

$$
\left.h^{*}(f) \geqslant \lim _{n \rightarrow \infty} h\left(\left.f\right|_{Y_{n}^{*}}\right)-h\left(\left.f\right|_{Y}\right) \quad \text { (we assume } \infty-\infty=0\right) .
$$

Proof. From Dinaburg's theorem it follows that for any $n$ there exists a measure $\mu_{n} \in \mathfrak{M}(X, f)$ such that $h_{\mu_{n}}(f) \geqslant h\left(\left.f\right|_{X_{n}}\right)-1 / n$ and supp $\mu_{n}$ $\subset Y_{n}$. Let $\mu \in \mathfrak{M}(X, f)$ be a cluster point of the set $\left\{\mu_{n}\right\}_{n=1}^{\infty}$. We have

$$
\mu(X \backslash Y)=\mu\left(\bigcup_{n=1}^{\infty}\left(X \backslash U_{n}\right)\right)=\lim _{n \rightarrow \infty} \mu\left(X \backslash U_{n}\right) .
$$

But, for $n$ fixed, $\mu_{k}\left(X \backslash Y_{n}\right)=0$ for almost every $k$, and therefore $\mu\left(X \backslash U_{n}\right)$ $=0$. Therefore, $\operatorname{supp} \mu \subset Y$. But in view of Goodwyn's theorem we have $h_{\mu}(f) \leqslant h\left(\left.f\right|_{Y}\right)$. Applying Theorem 4.2 , we obtain:

$$
\begin{aligned}
h_{\mu}^{*}(f) & \geqslant \liminf _{n \rightarrow \infty} h_{\mu_{n}}(f)-h_{\mu}(f) \\
& \geqslant \lim _{n \rightarrow \infty}\left[\dot{h}\left(\left.f\right|_{Y_{n}}\right)-1 / n\right]-h\left(\left.f\right|_{Y}\right)=\lim _{n \rightarrow \infty} h\left(\left.f\right|_{Y_{n}}\right)-h\left(\left.f\right|_{Y}\right) .
\end{aligned}
$$

Remark 4.2. If $X$ is a metric space, then the assumption of the existence of the sets $U_{n}$ is fulfilled automatically; it suffices to put $U_{n}$ $=\bigcup_{x \in Y_{n}} \operatorname{Int} B_{1 / n}(x)$.

One can apply the abore proposition in order to compute the topological conditional entropy in the examples from [12], [15]. The conditional entropy in these examples turns out to be equal to the usual topological entropy.

§ 5. Flows. Now let us consider a continuous flow $\left\{\varphi^{t}\right\}_{t \in \boldsymbol{R}}$ on a nonempty compact Hausdorff space $X$. We can write, as in the discrete case, $A_{\varphi}^{T}=\bigvee_{t \in[0, T)} p^{-t} A$ for $A \epsilon \mathfrak{A}, T>0$. The application of this notion is based on the following proposition:

Proposimion 5.1. For $A \in \mathfrak{A}, T>0$, there exists a $B \in \mathfrak{A}$ such that $B \geqslant A^{T}$.

Proof. Let $L \in \mathfrak{N}$ be a Lebesgue number for $A$. Let $\pi: X \times X \times$ $\times[0, T] \rightarrow X \times X$ be the natural projection; let $\Phi: X \times X \times[0, T] \rightarrow X \times X$ be the mapping defined as follows: $\Phi(x, y, t)=\left(p^{t} x, p^{t} y\right)$. Clearly, $\Phi$ is continuous. Write $Z=\pi\left(\Phi^{-1}(X \times X \backslash L)\right)$. $Z$ is compact and symmetric. If there exists a point $x \in X$ with $(x, x) \in Z$, then there exists a number $t \epsilon[0, T]$ such that $\left(\varphi^{t} x, \varphi^{t} x\right) \epsilon X \times X \backslash L$, but this is impossible. Fence $U=X \times X \backslash Z$ is an element of $\mathfrak{X}$. For any $(x, y) \epsilon U$ and $t \in[0, T]$ we have $\left(\varphi^{t} x, \varphi^{t} y\right) \in L$; therefore any finite cover $B$ chosen from the open cover $\{\{y:(x, y) \in U\}\}_{x \in \Phi}$ is a refinement of $A_{p}^{T}$.
In view of the above proposition, for $A \epsilon \mathfrak{A}$ and $T>0, A_{\varphi}^{T}$ belongs to $\mathscr{P}$. For $A, B \in \mathfrak{A}$ the function $\log N\left(A_{\varphi}^{T^{T}} \mid B_{\varphi}^{T}\right)$ is, as in the discrete ase, subaditive; therefore there exists a limit

$$
\lim _{x^{\prime} \rightarrow \infty} \frac{1}{T} \log N\left(A_{p}^{T} \mid B_{p}^{T}\right)=h_{\text {flow }}(\varphi, A \mid B) .
$$

Tinther, in the same way as in the discrete case, we obtain

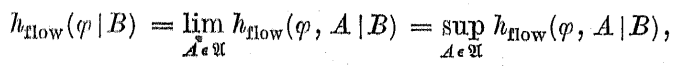

$$
\begin{aligned}
& h_{\text {llow }}^{*}(p)=\lim _{B \in \mathfrak{Q}} h_{\text {flow }}(p \mid B)=\inf _{\mathcal{B} \in \mathfrak{Q}} h_{\text {flow }}(p \mid B), \\
& h_{\text {flow }}(p)=h_{\text {llow }}(p \mid \Theta) \text {. }
\end{aligned}
$$

ThгоRтм 5.1. Tet $\left\{\phi^{l}\right\}_{\ell \in \boldsymbol{n}}$ be a continuous flow on a compact Hausdorff space $X$. Then for $T \in \boldsymbol{R}$ :

$$
\begin{aligned}
h\left(\varphi^{T}\right) & =|T| \cdot h_{\text {flow }}(\varphi), \\
h^{*}\left(p^{T}\right) & =|T| \cdot h_{\text {flow }}^{*}(\varphi) .
\end{aligned}
$$

Proof. We may assume that $T>0$; for $T=0$ the theorem is obvious; for $T<0$ it is a consequence of the case $T>0$ and the formulas $h(f)=h\left(f^{-1}\right)$ and $h^{*}(f)=h^{*}\left(f^{-1}\right)$.

Let $A, B, O, D \in \mathfrak{A}, B \geqslant A_{\varphi}^{T}, D \geqslant O_{\varphi}^{T}$. For $n \in \boldsymbol{N}$ we have

$$
N\left(\left(A_{p}^{T}\right)_{p}^{n}{ }_{p}^{n} \mid D_{p^{T}}^{n}\right) \leqslant N\left(A_{\varphi}^{n T} \mid O_{p}^{n T}\right) \leqslant N\left(B_{p}^{n_{p}} T \mid\left(C_{\varphi}^{T}\right)_{\varphi}^{n_{p}} T\right) ;
$$

thus

$$
h_{l}\left(\psi^{T}, A_{p}^{T} \mid D\right) \leqslant T \cdot h_{\text {llow }}(p, A \mid C) \leqslant h\left(p^{T}, B \mid C_{p}^{T}\right) \leqslant h\left(p^{T} \mid O_{p}^{T}\right) .
$$

In view of Proposition 5.1 the family $\left\{A_{\omega}^{T}: A \in \mathfrak{A}\right\}$ is cofinal with $\mathfrak{A}$; therefore we can take the limit with respect to $A$. We obtain

$$
\left.h(\varphi)^{T} \mid D\right) \leqslant T \cdot h_{\text {flow }}(\varphi \mid C) \leqslant h\left(\varphi^{T} \mid O_{\varphi}^{T}\right) \quad \text { for } \quad D \geqslant C_{\varphi}^{T} .
$$

Putting $\sigma=D=\Theta$, we obtain $h\left(\varphi^{T}\right) \leqslant T \cdot h_{\text {flow }}(\varphi) \leqslant h\left(p^{T}\right)$, whence (a) follows.

Taking in (5.1) the limit with respect to $D$, we obtain $h^{*}\left(\varphi^{T}\right)$ $\leqslant T \cdot h_{\text {mlow }}(p \mid O) \leqslant h\left(\phi^{q^{\prime}} \mid O_{p}^{t^{\prime}}\right)$, and $(\mathrm{b})$ follows, because $C$ is arbitrary.

OoRorilaky 6.1. Under the assumptions of the theorem

$$
\begin{aligned}
h\left(\phi^{T}\right) & =|T| \cdot h\left(\phi^{1}\right), \\
h^{*}\left(\phi^{T}\right) & =|T| \cdot h^{*}\left(\phi^{1}\right) .
\end{aligned}
$$

Remark 5.1. The part (a) of Oorollary 5.1 has already been known, but it has been proved only for metric spaces (Dinaburg [8], Bowen [4]). 
Remark 5.2. In the whole of Section 5 (except the case $T<0$ in Theorem 5.1 and in Corollary 5.1) all the theorems are valid for a oneparameter semigroup $\left\{\varphi^{t}\right\}_{l \geqslant 0}$ of continuous transformations of a space $X$ into itself (a semiflow).

\$ 6. Counterexamples. We have proved some properties of topological conditional entropy which are similar to the properties of usual topological entropy. In this part we shall give examples which show that some other properties of this type are not valid.

In the whole of Section 6 the symbols for spaces and transformations will be fixed.

Example 6.1. Let us consider the product of a countable number: of copies of the compact discrete two-element topological group $\boldsymbol{Z}_{2}$ : $Q=I \prod_{-\infty}^{\infty} \mathbf{Z}_{2} \cdot Q$ is a metric compact group. The shift, given by $T\left(x_{i}\right)_{i=\cdots}^{\infty} \ldots \infty$ $=\left(y_{i}\right)_{i=-\infty}^{\infty}, y_{i}=x_{i+1}$, is a continuous transformation $T: Q \rightarrow Q$. It is well known that $T$ is expansive and $h(T)=\log 2$.

Let $P=\{0\} \cup\{1 / n\}_{n=1}^{\infty} \subset \boldsymbol{R} . P$ is a compact metric space. Take $X=P \times Q ; f=\operatorname{id}_{P} \times T . f: X \rightarrow X$ is a homeomorphism. We have $h(f)$ $=\log 2$ and $f$ is $h$-expansive (from the proof of Theorem 3.4 it follows that the product of two $h$-expansive transformations is also $h$-expansive).

Denote by $Y$ the space obtained by identifying all the points of $X$ with the first coordinate $0 . Y$ is a compact metrizable space. $\{0\} \times Q$ is a subset of $X$ strictly invariant with respect to $f$, and therefore there exists exactly one homeomorphism $g: Y \rightarrow Y$ such that $\pi \circ f=g \circ \pi$, where $\pi: X \rightarrow Y$ is the natural projection. We have $h(g) \leqslant h(f)=\log 2$. Besides, $\left.g\right|_{\{1 / n\} \times Q}$ is conjugate in a natural way with $T$. Hence $h(g)=\log 2$. In view of Proposition 4.3 and Remark 4.2 we have also $h^{*}(g)=\log 2$. Thus we see that a factor of an $h$-expansive transformation may have positive conditional entropy.

It is not a very surprising result, because the image of an open cover: needs not be open (in fact, in the above example, if for some $A \in \mathfrak{A}(X)$ we have $h(f \mid A)=0$, then $\pi(A)$ is not open). But, as the next example shows, even the assumption that a projection from $X$ onto $Y$ is open is not sufficient.

ExAMPLe 6.2. Let $p_{n}: Q \rightarrow Q(n=1,2, \ldots)$ be defined as follows: $p_{n}\left(x_{i}\right)_{i=-\infty}^{\infty}=\left(y_{i}\right)_{i=-\infty}^{\infty}, y_{i}=\sum_{j=i-n}^{i+n} x_{j}$ (addition is in $Z_{2}$ ). The following properties of $p_{n}$ are easy to check:

(6.1) $p_{n}$ is continuous,

(6.2) for any oylinder (i.e., a set $C_{B_{-k}, \ldots, s_{i b}}=\left\{\left(x_{i}\right)_{i=-\infty}^{\infty} \in Q: x_{j}=\varepsilon_{j}\right.$ for $|j| \leqslant k\})$ we have $p_{n} O_{e_{-k}, \ldots, \varepsilon_{k}}=Q$ if $k<n$,
(6.3) $\quad p_{n} \circ T=T \circ p_{n}$

Now lot us define $p: X \rightarrow Y$ as follows: $p(0, x)=0 ; p(1 / n, x)$ $=\left(1 / n, p_{n}(x), n=1,2, \ldots\right.$ From properties $(6.1)-(6.3)$ we obtain:

(6.4) $p$ is continuous,

(6.5) $p$ is a surjection,

(6.6) $\quad p \circ f=g \circ p$,

(6.7) $p$ is an open mapping.

The properties (6.4)--(6.6) are obvious. To prove (6.7) notice that the family of all the sets of the form $a \times C_{b_{\ldots}, \ldots, b_{b}} \subset X$, where $a$ is open in $P$, is an open base for $X$; the image of such a set under the mapping $p$ is, in view of $(6.2)$, a sot of the form.

$$
(b \times Q) \cup \bigcup_{j=1}^{r}\left\{\frac{1}{s_{j}}\right\} \times C_{e_{-k}, j}, \ldots, \varepsilon_{k j}, j,
$$

where $b$ is open in $P$.

If $k \circ \psi=k$, then we have $h(\psi)=\sup h\left(\left.\psi\right|_{k-1}-1_{\{y\}}\right)$ (Bowen [4]). The next example shows that the above theorem is not valid for $h^{*}$ instead of $h$.

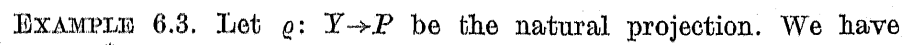
$\varrho \circ g=\varrho, h^{*}(g)=\log 2$, but for every $x \in P$ the transformation $\left.g\right|_{\ell}-I_{\{x\}}$ is expansive.

Now we shall show that the upper semi-continuity (even the constancy) of the measure-theoretic entropy does not imply asymptotical $h$-expansiveness of a transformation.

EXAMrex 6.4. Let $R \subset Q$ be a closed, $T$-invariant subset such that $\left.T\right|_{R}$ is uniquely ergodic and $h\left(\left.T\right|_{R}\right)=\gamma>0$ (see Hahn, Katznelson [13]). Fix a point $\omega=\left(\omega_{i}\right)_{i=-\infty}^{\infty} \in R$ and let $\omega^{n}=\left(\omega_{i}^{n}\right)_{i=-\infty}^{\infty}(i=1,2, \ldots)$ be defined by $\omega_{k n+j}^{n}=\omega_{j}$ for $j=0,1, \ldots, n-1, k \in Z$. Now define a closed $(T \times g)$-invariant set $Z$ by

$Z=\left\{(x, y) \in Q \times Y:(y=0, \infty \in R)\right.$ or $\left(y \in\left\{\frac{1}{n}\right\} \times R, x=T^{j} \omega^{n}\right.$ for some $\left.\left.j\right)\right\}$.

$\varphi=T \times\left. g\right|_{Z}$ is a homeomorphism of $Z$ onto itself. We shall compute the conditional entropy of $p$. The family of all open finite covers of $Z$ of the form.

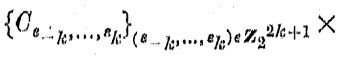

$$
\begin{aligned}
& \times\left.\pi\left[\{a \times R\} \cup\left(\left\{\left\{\frac{1}{i}\right\}\right\}_{i=1}^{m-1} \times\left\{O_{\varepsilon_{-j}, \ldots, \varepsilon_{j}}\right\}_{\left(\varepsilon_{-j}, \ldots, \varepsilon_{j}\right) \epsilon \mathbf{Z}_{2}{ }^{2 j+1}}\right)\right]\right|_{z},
\end{aligned}
$$


where $a=\{0\} \cup\{\mathcal{1} / i\}_{i=m}^{\infty} \subset P$, is cofinal with $\mathfrak{A}(Z)$ (remember that $\{\{1 / i\}\}_{i=1}^{m-1}$ is an open cover of $\left.\{1 / i)_{i=1}^{m-1}\right)$. But for a cover $B$ of the above form we have $\left\{\omega^{m}\right\} \times(\{1 / m\} \times R) \prec B^{n}$ for $n=1,2, \ldots$, and therefore

$$
\begin{aligned}
h(\varphi \mid B) & \geqslant h\left(\varphi,\left\{\omega^{m}\right\} \times\left(\left\{\frac{1}{m}\right\} \times R\right)\right)=\frac{1}{m} h\left(\gamma^{m},\left\{\omega^{m}\right\} \times\left(\left\{\frac{1}{m}\right\} \times R\right)\right) \\
& =\frac{1}{m} h\left(\left.\phi^{m}\right|_{\left.\left\{\omega^{m}\right\} \times(11 / m\} \times R\right)}\right)=\frac{1}{m} h\left(\left.T^{m}\right|_{R}\right)=h\left(\left.T\right|_{R}\right)=\gamma .
\end{aligned}
$$

It is easy to check that $h(\varphi)=\gamma$, and thus also $h^{*}(p)=\gamma$. Denote by $\mu_{0}$ the unique measure from $\mathfrak{M}(Z, \varphi)$ whose support is contained in $R \times\{0\}$, and by $\mu_{n}$ the unique measure from $\mathfrak{M}(Z, p)$ whose support is contained in $[Q \times(\{1 / n\} \times R)] \cap Z(n=1,2, \ldots)$. It is easy to see that they are well defined, ergodic, and that there are no more ergodic measures in $\mathfrak{M}(Z, p)$. Hence for any $\mu \in \mathfrak{M}(Z, \varphi)$ there exist numbers $q_{n} \geqslant 0, n=0,1,2, \ldots$, such that $\sum_{i=0}^{\infty} q_{i}=1$ and $\mu=\sum_{i=0}^{\infty} q_{i} \mu_{i}$. We have $h_{\mu}(p)=\gamma$, because $h_{\mu_{n}}(p)$ $=\gamma$ for $n=0,1,2, \ldots$

Remark 6.1. We can use the spaces and the transformations defined. in Example 6.4 to obtain a slightly stronger result than that of Example 6.2. Namely, the transformation $g$ with positive conditional entropy may be a factor even of an expansive transformation under an open mapping. Take a factor of $\varphi$ under $\mathrm{id}_{Q} \times \varrho$. It is expansive, but $i d_{P}$ is its factor under an open mapping.

The next example shows that it is possible for an inverse limit of expansive transformations not to be even asymptotically $h$-expansive.

EXAMPLE 6.5. Write $Y_{n}=\{1 / k\}_{k=1}^{n} \times Q \cup\{0\}, g_{n}: Y_{n} \rightarrow Y_{n},\left.g_{n}\right|_{\{1 / k)_{k=1}^{n} \times Q}$ $=\mathrm{id} \times T, g_{n} 0=0$. We have projections $\tau_{n m}: Y_{n} \rightarrow Y_{m}$ for $n>m$ defined as follows:

$$
\tau_{n m}\left(\frac{1}{k}, x\right)=\left\{\begin{array}{lll}
\left(\frac{1}{k}, x\right) & \text { for } & k \leqslant m \\
0 & \text { for } & 7>m,
\end{array}\right.
$$

$\tau_{n m} 0=0$. It is easy to see that $\left(Y_{n}, g_{n}\right)_{n=1}^{\infty}$ with mappings $\left(\tau_{n m}\right)_{n>m}$ form an inverse system with the limit $(Y, g)$. All $g_{n}$ 's are expansive, but $h^{*}(g)>0$.

§ 7. Cascades with a homogeneous measure. Bowen in [5] proved. that any continuous group endomorphism of a Lie group is $h$-expansive. We can see at once that this statement is not valid for an arbitrary compact group instead of a lie group, because no countable infinite product of transformations with positive entropy is $h$-expansive. But we shall show that every continuous group endomorphism of a compact group is asymptotically $h$-expansive if its entropy is finite. For this purpose we shall use the notion of a homogeneous measure.

Let $(X, f)$ be a cascade and let $\mu \in \mathfrak{M}(X)$. For $A \in \mathfrak{Q}$ write

$$
P(A)=\bigcup_{x \in X}\{a \in A: x \in a,[\underset{b \in A}{\forall}(x \in b \Rightarrow \mu(a) \geqslant \mu(b))]\} .
$$

Of course, $P(A) \in \mathfrak{Q}$. The measure $\mu$ is called f-homogeneous if there exist mappings $D(\cdot): \mathfrak{H} \rightarrow \mathfrak{A}$ and $o(\cdot): \mathfrak{A} \rightarrow(0, \infty)$ such that for any $E \in \mathfrak{A}$, $k \geqslant 1$, a $P\left(D^{h}\right), d \in D(H)^{k}$ we have

$$
\mu\left(d_{t}\right) \leqslant c(\mathbb{E}) \cdot \mu(a) .
$$

It is exsy to check that for $X$ being metric the above definition is equivalent to the definition given by Bowen [4].

For $A \in \mathfrak{A}$, let

$$
M_{l k}(A)=\max _{a \in A^{l^{k}}} \mu(a), \quad m_{l^{\prime}}(A)=\min _{a \in P\left(A^{k_{k}}\right)} \mu(a) .
$$

For $B \geqslant A$ we have $M_{l_{k}}(B) \leqslant M_{l_{l}}(A)$, and therefore for an $f$-homogeneous measure

(7.2) $M_{k}(D) \leqslant o(E) \cdot m_{k}(E) \quad$ for $\quad D, E \in \mathfrak{A}, D \geqslant D(E), k=1,2, \ldots$

Iismma 7.1. Let $A, B \in \mathfrak{A}, B \geqslant A$. Then

$$
N\left((\mathrm{St} B)^{k} \cdot A^{k_{i}}\right) \cdot m_{k c}(B) \leqslant M_{k i}(\mathrm{St} A) .
$$

Proof. Let $a$ be the element of $A^{k}$ for which the number $N\left(a, \operatorname{St}\left(B^{k}\right)\right)$ is the largest. In view of Lemma $3.3, \mathrm{St}\left(B^{k}\right) \geqslant(\mathrm{St} B)^{k}$, and so this number is not smaller than $p=N\left((\mathrm{St} B)^{k} \mid A^{k}\right)$. In view of Lemma 3.1 there exists a discover $C \subset P\left(B^{i c}\right)$ such that $o n a \neq \varnothing$ for $c \in C$ and that $a \subset \cup$ sst $\left(c, B^{k}\right)$ : $c \in C\}$ (because st $\left.\left(c, P\left(B^{k}\right)\right) \subset \operatorname{st}\left(c, B^{k}\right)\right)$. We have Card $\left\{\operatorname{st}\left(c, B^{k}\right): c \in C\right\}$ $\leqslant$ Card $O$, and therefore Card $O \geqslant p$. Let $a=\bigcap_{i=0}^{k-1} f^{-i} a_{i}, a_{i} \in A$. We have

$$
\begin{aligned}
& \cup\left(y \subset \operatorname{stc}\left(a, B^{/ h}\right)\right.
\end{aligned}
$$

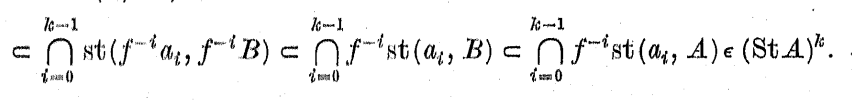

II.ence

$$
p \cdot m_{l_{k}}(B) \leqslant \mu(\cup O) \leqslant M_{l_{k}}(\mathrm{St} A) .
$$

Ticworwa 7.1. Let $(X, f)$ be a cascade, $h(f)<\infty$. If there exists an $f$-homiogeneous 'measure $\mu \in \mathfrak{M}(X)$, then $f$ is asymptotically h-expansive.

Proof. Putting in Lemma 7.1 $A=\Theta, B=E$, we obtain

$$
N\left((\mathrm{St} E)^{k}\right) \cdot m_{l k}(\mathbb{E}) \leqslant 1 \quad \text { for } \quad E \in \mathfrak{A} .
$$


The following inequality is obvious:

$$
1 \leqslant N\left(D^{l c}\right) \cdot M_{k_{k}}(D) \text { for } D \in \mathfrak{A} \text {. }
$$

Hence $M_{l_{c}}(D)>0$ for $D \in \mathfrak{A}$; from this and (7.2) it follows also that $m_{l k}(E)^{\circ}$ $>0$ for $E \in \mathfrak{U}$. Thus from (7.2) and (7.4) we obtain

$$
m_{k}(E) \geqslant \frac{1}{N\left(D^{k}\right) \cdot c(E)} \quad \text { for } \quad D, E \in \mathfrak{A}, D \geqslant D(E),
$$

and from (7.2) and (7.3) we obtain

$$
M_{l}(D) \leqslant \frac{c(\mathbb{E})}{N\left((\mathrm{St} E)^{k}\right)} \quad \text { for } \quad D, E \in \mathfrak{A}, D \geqslant D(\mathbb{H}) .
$$

Putting $E=B$ in (7.5) and $D=\operatorname{St} A$ in (7.6) and applying Lemma 7.1 to them, we get

(7.7). $\quad N\left((\mathrm{St} B)^{k} \mid A^{k}\right) \leqslant \frac{c(E)}{N\left((\mathrm{St} E)^{k}\right) !} \cdot N\left(D^{k}\right) \cdot c(B)$

for $\quad A, B, D, E \in \mathfrak{A}$, St $A \geqslant D(E), D \geqslant D(B), B \geqslant A$.

Hence

(7.8) $\quad h(f, \mathrm{St} B \mid A) \leqslant h(f, D)-h(f, \mathrm{St} E)$

for $\quad A, B, D, E \in \mathfrak{A}$, St $A \geqslant D(E), D \geqslant D(B), B \geqslant A$.

We may take the limit with respect to $D$ :

(7.9) $\quad h(f, \operatorname{St} B \mid A) \leqslant h(f)-h(f, \operatorname{St} \boldsymbol{E})$

$$
\text { for } \quad A, B, E \in \mathfrak{A}, \text { St } A \geqslant D(E), B \geqslant A \text {. }
$$

Now we take the limit with respect to $B$, applying Proposition 3.5 :

(7.10) $\quad h(f \mid A) \leqslant h(f)-h(f$, St $E)$ for $\quad A, E \in \mathfrak{A}$, St $A \geqslant D(E)$.

Further, take the limit with respect to $A$ :

(7.11) $\quad h^{*}(f) \leqslant h(f)-h(f, \mathrm{St}, E) \quad$ for $\quad$ He $\mathfrak{A}$.

Finally, taking the limit with respect to $E$ and applying Proposition 3.5 once more, we obtain (notice that here we use the assumption that $h(f)<\infty)$ :

$$
h^{*}(f) \leqslant 0 .
$$

The assumptions of Theorem 7.1 are difficult to check directly. But they are a consequence of certain other conditions, pointed out below, which admit a much simpler verification.
Let $G$ be a group. Let $\Phi$ be a (left-hand) action of $G$ on a space $X$ (i.e., a homomorphism of $G$ into the group of all homeomorphisms of $X$ ). We shall write $g \cdot x=(\Phi g) x$ for $g \in G, x \in X$. An action is called transitive if $\underset{n:, y \in X, f \in G t}{\forall} g \cdot x=y$. An action is called equicontinuous if the family $\Phi(G)$

of. homeomorphisms of $X$ onto itself is equicontinuous, i.e., for any $V \in \mathfrak{N}(X)$ there existis a $W \in \mathfrak{N}(X)$ such that if $g \in G$ and $(x, y) \in W$, then $(g \cdot x, g \cdot y) \in V$. A measure $\mu \in \mathfrak{M}(X)$ is called invariant with respect to an action $\Phi$ if $\mu \in \bigcap_{\| \in G} \mathfrak{M}(X, \Phi g)$.

TIISORHM 7.2. Let $(X, f)$ be a cascade. Let $G$ be a group and $T: G \rightarrow G$ a homomorphism. Hurther, let $\Phi$ be a transitive equicontinuous action of $G$ on $X$ such that $f(g \cdot x)=T g \cdot f x$ for $x \in X, g \in G$. Let a measure $\mu \epsilon \mathfrak{M}(X)$ be invariant with respeat to $\Phi$. Then $\mu$ is f-homogeneous.

Proot. Note that

(7.13) $\quad f^{n}(g \cdot x)=I^{n} g \cdot f^{n} \cdot x$ for $g \in G, x \in X, n=0,1, \ldots$

Liet $g \in G, X \subset X, n \in N$. We have

$$
g \cdot\left(f^{-n} X\right)=\left\{x: f^{n}\left(g^{-1} \cdot x\right) \in Y\right\}=\left\{x: T^{n} g^{-1} \cdot f^{n} x \in Y\right\}=f^{-n}\left(T^{n} g \cdot Y\right) .
$$

\section{Hence}

(7.1.4) $\quad y \cdot\left(f^{\cdots n} Y\right)=f^{\cdots n}\left(I^{n} g \cdot Y\right)$ for $\quad g \in G, Y \subset X, n=0,1, \ldots$

Now tiake $E \in \mathfrak{A}$. The action $\Phi$ is equicontinuous, and therefore there exists an open non-emptiy subset $U$ of $X$ such that the set $\{(x, y) \in X \times X$ : $\exists x, y \in g \cdot U\}$ is contained in some Lebesgue number of $E$. Write

$$
U_{x:}=\{y \in X: \exists x, y \in g \cdot U\}, \quad U_{x}^{n}=\bigcap_{i=0}^{n-1} f^{-i} U_{f^{i} x}
$$

fol $x \in X, w=1,2, \ldots$ From the definition of $U_{x}$ it follows that $U_{x} \prec E$ for $x \in X$. For $x \in X$ and $k \in G$ we have

$$
k \cdot U_{x}=\left\{y \in X: \underset{g \in G}{\exists} x, k^{-1} \cdot y \in g \cdot U\right\}=\{z \in X: \underset{g \in G}{\exists k \cdot x, z \in k g \cdot U\} .}
$$

But the left-hand side multiplication by $z_{0}$ is an isomorphism of $G$ onto itself, and therefore we obtain.

$$
\psi \cdot U_{x}=V_{k \cdot x} \text { for } x \in X, k \in G \text {. }
$$

In viow of $(7.1 .3)-(7.1 .5)$, for $i=0, \ldots, n-1$ we have

$$
g \cdot f^{-i} U_{f i x}=f^{-i}\left(T^{i} g \cdot U_{f^{i} x}\right)=f^{-i}\left(U_{T^{i}{ }^{i} \cdot f^{i} x}\right)=f^{-i} U_{f^{i}\left(g^{\prime} x\right)},
$$

and thus

$$
g \cdot U_{x}^{n}=U_{g \cdot x}^{n} \quad \text { for } \quad x \in X, g \in G, n=1,2, \ldots
$$


Now we choose a finite cover $D(E)$ from the open cover $\{g \cdot U\}_{\text {ged }}$ of $X$. Let $x \in d \in(D(E))^{n}$. Then $x \in d=\bigcap_{i=0}^{n-1} f^{-i}\left(g_{i} \cdot U\right)$ for some $g_{0}, \ldots ; g_{n-1} \in G$. Hence $f^{i} x \in g_{i} \cdot U$, and therefore $g_{i} \cdot U \subset U_{f^{i}, x}$ for $i=0, \ldots, n-1$. Oonsequently, $d \subset U_{x}^{n}$. Thus we have

(7.17) $\mu(d) \leqslant \mu\left(U_{x}^{n}\right)$ if only $x \in d \epsilon(D(E))^{n}(n=1,2, \ldots)$.

Now fix $x \in X$. We have $U_{f^{i} x} \subset a_{i}$ for some $a_{i} \in t, i=0, \ldots, n, \ldots$. Hence $x \in U_{x}^{n} \subset \bigcap_{i=0}^{n-1} f^{-i} a_{i} \in \mathbb{E}^{n}$, and so

(7.18) $\mu\left(U_{x}^{n}\right) \leqslant \max \left\{\mu(a): x \in a \in E^{n}\right\} \quad$ for $\quad x \in X, \|=1,2, \ldots$

The measure $\mu$ is invariant with respect to $\Phi$ and $\Phi$ is transitive; thus from (7.16) it follows that

$$
\mu\left(U_{x}^{n}\right)=\mu\left(U_{y}^{n}\right) \quad \text { for } \quad x, y \in X, n=1,2, \ldots
$$

In view of $(7.17)-(7.19)$ the measure $\mu$ is $f$-homogeneous (put $c(E)=1$ ).

Evidently the above theorem is also valid for a right-hand action of a group.

Now we shall show the simplest examples of transformations with finite entropy for which the assumptions of Theorem 7.2 are fulfilled.

ExAMPLE 7.1. Let $G$ be a compact group and $f: G \rightarrow Q$ a continuous homomorphism, $h(f)<\infty$. Put in Theorem $7.2 X=G, T=f, \mu$ the Haar measure of $G$, and let the action be right side multiplication. Then the assumptions are fulfilled. Hence $f$ is asymptotically $h$-expansive. If we take $f x=T x \cdot g_{0}\left(g_{0}\right.$ is a fixed element of $\left.G\right)$, i.e., an affine transformation, then the assumptions of Theorem 7.2 are also fulfilled.

EXAMPLE 7.2. Let $G$ be a locally compact group with a closed subgroup $H$ such that $X=G / H$ is compact and there exists a $G$-imvariant normed measure on $X$. Then a continuous homomorphism $T: G \rightarrow G$ which preserves $H$ induces a continuous transformation $f: X \rightarrow X$. The action of $G$ is natural. We assume also $h(f)<\infty$. All the assmumptions of Theorem 7.2 are very easy to check, except, maybe, the equicontinuity of the action. But the natural projection $Q \rightarrow Q / H$ is uniformly continuous and the superposition of a uniformly continuous mapping with an equicontinuous family of mappings gives an equicontimuous family of mappings. Hence the transformation $f$ defined above is asymptotically
$h$-expansive.

§ 8. Connection with pressure. Let $(X, f)$ be a cascade and let $p$ : $\boldsymbol{X} \rightarrow \boldsymbol{R}$ be a continuous function. The notion of topological entropy may be generalized to the notion of pressure $P(f, p)$ (see $[17]$ ).
The following facts are stated in [17]:

$$
P(f, 0)=h(f) \text { and } \quad P(f, \varphi)=\sup _{\mu \in \mathfrak{M}(x, f)}\left[h_{\mu}(f)+\int \varphi d \mu\right] .
$$

If for some $\mu_{0} \in \mathfrak{M}(X, f)$, the equality $P(f, \varphi)=h_{\mu_{0}}(f)+\int \phi \lambda \mu_{0}$ holds, then $\mu_{0}$ is called an equilibrium state for $(f, \varphi)$. If $h(f)$ is an upper semicontinuous function, then

$$
h_{\mu}(f)=\inf _{p \in \mathcal{C}(X, \boldsymbol{n})}\left[P(f, \phi)-\int \psi d \mu\right]
$$

Thus Theorem 4.2 yields the tollowing

ConoliaAm 8.1. If $f$ is asymptotically h-expansive, then there exists an equilibrium state for $\left(f, \psi^{\prime}\right)$ for every $p \in O(X, \boldsymbol{R})$ and the formula (8.1) is valid for every measure $\mu \in \mathfrak{M}(X, f)$.

In Section 7 we have given some important examples in which the assumptions of this corollary are fulfilled.

Wo may remark that it is easy to answer the question, raised in [17], whether the formula (8.1) is always valid. The right-hand side of (8.1) regarded as a function of $\mu$ is an infimum of a family of continuous functions, and therefore it is upper semicontinuous. Hence (8.1) is valid for every $\mu \in \mathfrak{M}(X, f)$ iff $h(f)$ is an upper semicontinuous function.

\section{Reference}

[1] R. T. Adler, A. (4. Kon heim, M. H. McAndrow, Topological entropy, Trans. Amor. Math. Soc. 114: (1965), pp. 309-319.

[2] P. Billingsloy, Ergodic theory and information, Wiley, New York 1965.

[3] R. Bowen, Topologieal entropy and Axiom A, Proc. Symp. Pure Math., Vol. 14, pp. $23-41$.

[4] - Lintropy for group endomorphisms and homogeneous spaces, Trans. Amer. Math. Soc. 153 (1971), pp. 4:01-414.

[5] - Intropy-expansive maps, ibid. 164 (1972), pp. 323-333.

[0] -... Topological entropy for noncompact sets, ibid. 184 (1973), pp. 125-136.

[7] M. Donkar, Wne démonstration nouvolle da theorème de Goodwyn, C. R. Acad. Sci. Paris 275 (1972), pp. 735-738.

[9] H. T. Dinaburg, A sonnection between various entropy aharacterizations of dynamionl syslems. (Rumian), Izy. Akad. Nauk SSSR, Ser. Mat. 35 (1971), pp. $324-3363$.

[0] T. N. T. Goodmun, Relaling topological entropy and measure entropy, Bull. Jondon Math. Soc. 3 (1971), pp. 176-180

[10] I. W. Goodwyn, 'T'opological entropy bounds measure-theoretio entropy, Proc. Amer. Math. Soc. 23 (1969), pp. 679-688.

[11] - The product theorem for topological entropy, Trans. Amer. Math. Soc., 158 (1.971), pp. 44:5 4.62. 
[12] B. M. Gureviê, Topological entropy of denumerable Marloov ohains, Dokl. Akad. Nank SSSR, 187 (1969), pp. 715-718. (Soviet Math. Dokl. 10 (1969), pp. 91.1915)

[13] F. Hahn, Y. Katznelson, On the entropy of uniquely ergodic transformalions, Trans. Amer. Math. Soc., 126 (1967), pp. 335-360.

[14] J. L. Kelley, General topology, Van Nostrand, Princetion 1955.

[15] M. Misiurewicz, Diffeomorphism without any measure with maximal entropy, Bull. Acad. Polon. Sci., Sér. sci. math., astr. et phys. 21 (1973), pp. 903-910.

[16] M. Smorodinsky, Ergodic theory, entropy, Luet. Notes in Math. 214, Springer, Berlin 1971.

[17] P. WaIters, A variational prinaiple for the pressure of continuous trans formations, preprint.

WARSAW UNTVERSITY

INSTITUTE OF MATHEMATITCS

\section{A reflexive Banach space which is not sufficiently Euclidean}

by

\author{
WHLIA A M. JOHNSON* (Columbus)
}

Alstract. An example is given of a reflexive Banach space with uneonditional basis which is not sufficiontly Euolidean.

I. Introduction. In [8], Stegall and Retherford ask whether every reflexive Banach space $Y$ is sufficientily Euclidean; i.e., whether $Y$ contains a sequence $\left(E_{n}\right)$ of subspaces with $\sup d\left(E_{n}, l_{2}^{n}\right)<\infty$ for which there are projections $P_{n}$ of $X$ onto $E_{n}$ satisfying $\sup \left\|P_{n}\right\|<\infty$. $(d(E, F)$ is the Banach-Mazur distance coefficient inf $\left\{\|T\|\left\|T^{-1}\right\|: T\right.$ is an isomorphism from $D$ onto $I\}$.) This problem has a negative answer. In fact, we construct a rellexive Banach space $Y$ with unconditionally monotone basis for which $\|P\| \geqslant 2^{-6} d\left(W, l_{2}^{n}\right)^{-2} n^{\mathrm{x} / 2}$ for any projection $P$ from $Y$ ontio an widimensional subspace $W$.

We ase standard Banach space theory notation as may be found e.g. in $[6]$. We would like to thank Professor T. Figiel for simplifying the proof that the example constructed in Section II is reflexive.

II. The example. We work with the space $X$ of sequences of scalars which have only finitely many non-zero coordinates. Given a set $E$ of integers and $x \in X, E x$ is the sequence which agrees with $x$ in coordinates in $E$ and is zero in the other coordinates. A sequence $\left(E_{i}\right)_{i=1}^{n}$ of sets of positive integers is called allowable provided $E_{i} \cap E_{j}=\emptyset$ for $i \neq j$ and $t_{i} \subseteq[n+1, \infty)$ for $1 \leqslant i \leqslant n$. We will construct a norm $\|\cdot\|$ on $X$ for which the unit vectors $\left(e_{n}\right)$ form an unconditionally monotone basis so that the completion of $(X,\|\cdot\|)$ is reflexive and $X$ satisfies

(*) $\quad\|x\|=\max \left(\|x\|_{c_{0}}, \frac{1}{2} \sup \left\{\sum_{i=1}^{n}\left\|E_{i} \infty\right\|:\left(E_{i}\right)_{i=11}^{n}\right.\right.$ is allowable $\left.\}\right)$.

All Huclidean subspaces of $Y=\left[e_{n(n)}\right]$ are badly complemented if $X$ satisfies (*) and $(p(n))$ grows tast enough. The proof of this assertion makes use of the tollowing proposition, whose proot is omitted because it involves only a nonessential modification of the argument for Theorem $A$ in [7] and a stiandard perturbation argument.

* Supported by NSF GP--33578. 